\title{
An assembly gap control method based on posture alignment of wing panels in aircraft assembly

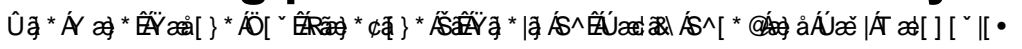

\begin{abstract}
Purpose - The gaps between two mating surfaces should be strictly controlled in precision manufacturing. Oversizing of gaps will decrease the dimensional accuracy and may reduce the fatigue life of a mechanical product. In order to reduce the gaps and keep them within tolerance, the relative posture (orientation and position) of two components should be optimized in the assembly process. This paper presents an optimal posture evaluation model to control the assembly gaps in aircraft wing assembly.

Design/methodology/approach - Based on the step alignment strategy, i.e. preliminary alignment and refined alignment, the concept of a small posture transformation (SPT) is introduced. In the preliminary alignment, an initial posture is estimated by a set of auxiliary locating points (ALPs), with which the components can be quickly aligned near each other. In the refined alignment, the assembly gaps are calculated and the formulation of the gaps with component posture is derived by the SPT. A comprehensive weighted minimization model with gap tolerance constraints is established for redistributing the gaps in multi-regions. Powell-Hestenes-Rockafellar (PHR) optimization, Singular Value Decomposition (SVD) and KD-tree searching are introduced for the solution of the optimal posture for localization.

Findings - Using the SPT, the trigonometric posture transformation is linearized, which benefits the iterative solution process. Through the constrained model, overall gaps are minimized and excess gaps are controlled within tolerance.

Practical implications - This method has been tested with simulated model data and real product data, the results of which have shown efficient coordination of mating components.

Original/Value - This paper proposed an optimal posture evaluation method for minimizing the gaps between mating surfaces through component adjustments. This will promote the assembly automation and variation control in aircraft wing assembly.
\end{abstract}

Keywords Mating surfaces; assembly gaps; gap tolerance; posture evaluation; wing panel; assembly automation Paper type Research paper

\section{Introduction}

The wings are the principal components of an aircraft, generating lift force and containing the fuel tanks. Their outer shape accuracy and inner leakproofness are important for aerodynamic performance, structure strength and safety. In the manufacturing process, especially the assembly process, the dimension, shape and position of the wing components are highly specified by the aerospace manufacturers. However, due to unavoidable factors (e.g. gravity, assembly stress, and environment), gaps or interferences may occur between the mating surfaces in the assembly process of a wing panel and its understructure. The popular application of composite materials in commercial and military aircraft increases the severity of the problem, since the dimension and shape accuracy of a composite wing structure are lower than an alloy-based structure. In order to minimize the assembly gaps and keep them within tolerance, the relative posture of the wing components should be well aligned. In automated aircraft assembly system, the wing components are positioned and adjusted by numerically controlled locators (NCLs) with high location accuracy. Thus, how to evaluate an optimal posture of a component from measured 3D values is a primary problem in the automation assembly process, since it should provide a target to plan a moving path for the NCLs to adjust the component.

From a mathematical point of view, posture evaluation can be considered as an optimal matching of two point sets, which has been widely studied in medical imaging, computer vision and reverse engineering. Several algorithms have been developed and widely used, such as Singular Value Decomposition (SVD) (Arun et al., 1987), 
Unit Quaternions (Horn et al., 1988), Iterative Closest Point (ICP) method (Besl et al., 1992) and its derivatives (Gregory et al., 2002; Andrew et al., 2003; Almhdie et al., 2006). In the field of aircraft manufacture, a series of posture evaluation methods based on engineering constraints have been developed to meet assembly requirements. Felus et al. (2005) presented an evaluation model with random errors to improve accuracy in coordinate transformation. Yu et al. (2010) proposed a matching algorithm with geometry constraints, which guarantees that the selected points remain collinear, symmetric or co-planar after a rigid transformation. Chen et al. (2015) presented a best-fitting model with position uncertainty to ensure the validity and accuracy of the calculated results. For large components assembly, Cheng et al. (2014) presented a new method to update the nominal coordinates of key measurement points by introducing a thermal deformation factor, and thus improved the posture accuracy. Zheng et al. (2013) proposed an evaluation algorithm based on a synthetic error including position and coaxiality errors, and they increased the assembly quality for a spacecraft module.

In summary, the previous researches have presented solutions to the modeling and optimization of accurate posture evaluation, but they are dedicated to special applications, and the target error functions are dependent on the 3D deviations of limited corresponding points. These methods are unsuitable for the assembly of a wing panel and its understructure. Firstly, it is difficult to find the key measurement points to represent the wing panel; secondly, the deformation caused by gravity and manufacture makes the panel difficult to fit well with the understructure, which finally results in assembly gaps (Wilma et al., 2007; Jamshidi et al., 2010; Smith, 2011).

Assembly gaps reduce the integrity of mechanical structures and may result in poor dynamic performance. In order to control the gaps between mating surfaces, a pre-assembly method is commonly used in a conventional wing assembly line. When the understructure is fixed as a reference on the assembly tooling, the wing panel is pre-joined with it, and the gaps are measured using feeler gauges and the edge alignment is checked visually. Then, the wing posture is adjusted manually by the crane according to measurement results. The iterative process of measurement-adjustment-measurement is continued until the gaps meet the assembly requirements. This method relies on the operator's experience and judgment, so it is subjective and not repeatable (Qian et al., 2005). When the wing's inner shape is complex and the actual gaps are very close to the lower tolerance boundary, it is very difficult for an operator to coordinate and relocate the panel.

In order to solve the control and coordination problem of assembly gaps in wing assembly, an optimal posture evaluation model is proposed in this paper, based on which the gaps can be digitally controlled and redistributed. A step alignment strategy, i.e. preliminary alignment and refined alignment, is introduced. In the preliminary alignment, an initial posture is estimated by a set of auxiliary locating points (ALPs). In the refined alignment, the definition of assembly gap is given, and the formulation of the gap with respect to a Small Posture Transformation (SPT) is derived, which helps to updates the new gap without exhaustive searching. Accordingly, a comprehensive evaluation model is established with gap tolerance constraints. The Powell-Hestenes-Rockafellar (PHR) optimization algorithm, SVD algorithm, and KD-tree algorithm are adopted to solve the optimal model to obtain transformation matrix and posture parameters. Finally, examples with simulated model data and actual product data are given to illustrate the validity of the proposed approach.

\section{Automated assembly of aircraft wing}

\section{Automated assembly process:}

An aircraft wing is composed of a upper panel, a lower panel, and a middle understructure. The understructure is a skeleton structure made up of metal spars, frames, ribs etc., thus it has high strength and shape accuracy. In wing assembly, the understructure is taken as the datum component, with which the panel will adjust its posture to coordinate their assembly relationships during the assembly process. The adjustment of a panel is commonly automated by NCLs in an automated assembly system. The panel is first positioned on the NCLs, and then a laser tracker and scanner are used to obtain the mating surface data of the panel and understructure. According to the 
measurement data, the integration system calculates the optimal joining posture of the panel, by which a moving path is planned for the NCLs to adjust the panel in real-time. When the adjustment is finished, the panel posture, NCL status, environment parameters are stored in the assembly database, as shown in figure 1.

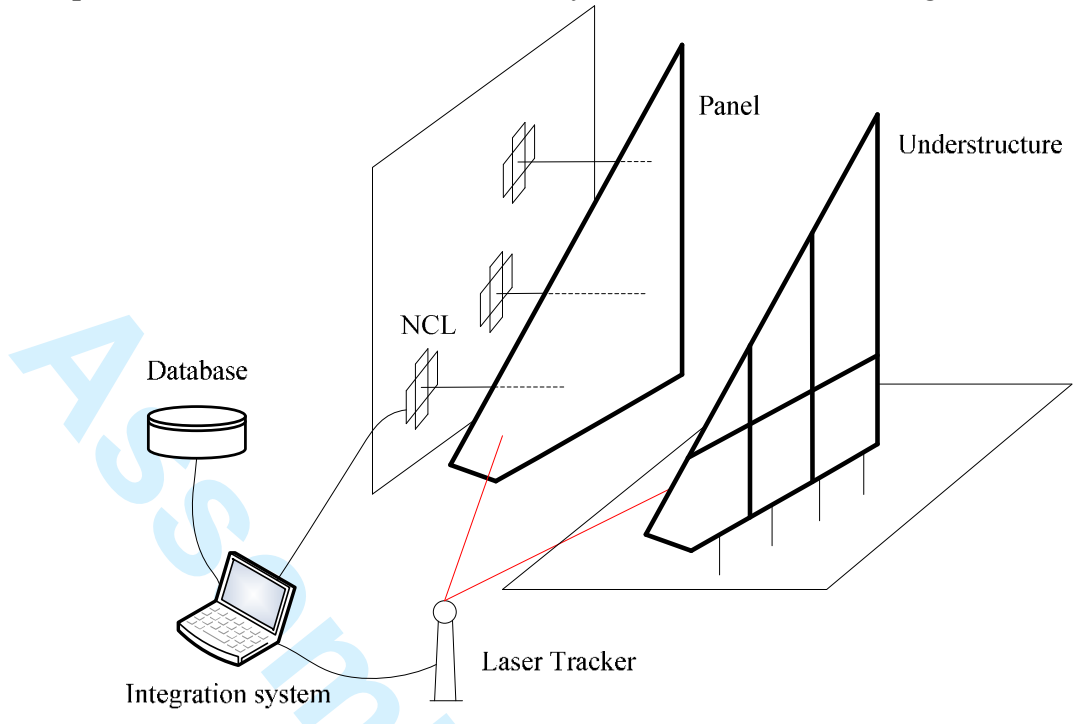

Figure 1 Automated assembly system of the wing

\section{Step alignment strategy :}

In order to keep a space for scanning measurement and guarantee the assembly safety, the panel is positioned far away from the understructure by the crane, so the assembly gaps are difficult to be directly calculated and controlled. In this paper, a step alignment strategy, i.e. preliminary alignment and refined alignment, is applied. In the preliminary alignment, a few corresponding points $(>3)$ of the components are used to align them efficiently without considering the assembly requirements. In the refined alignment, the gaps between the mating surfaces are first calculated; a slight posture adjustment is then applied to the panel to meet the gap constraints, as shown in figure 2 .

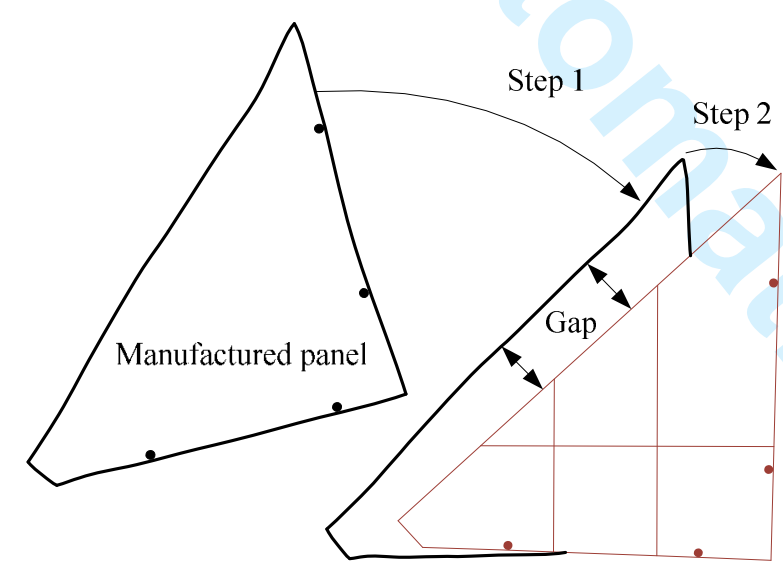

Figure 2 Step alignment of the panel

The advantage of a step alignment strategy embeds two aspects: (I) overcoming the problem of a lack of accurate locating points, where the refined alignment can improve the positioning accuracy; (II) the preliminary alignment offers a reasonable initial value for optimization in the refined alignment, which improves the stability of registration algorithm for two scanned surfaces.

\section{Small posture transformation}




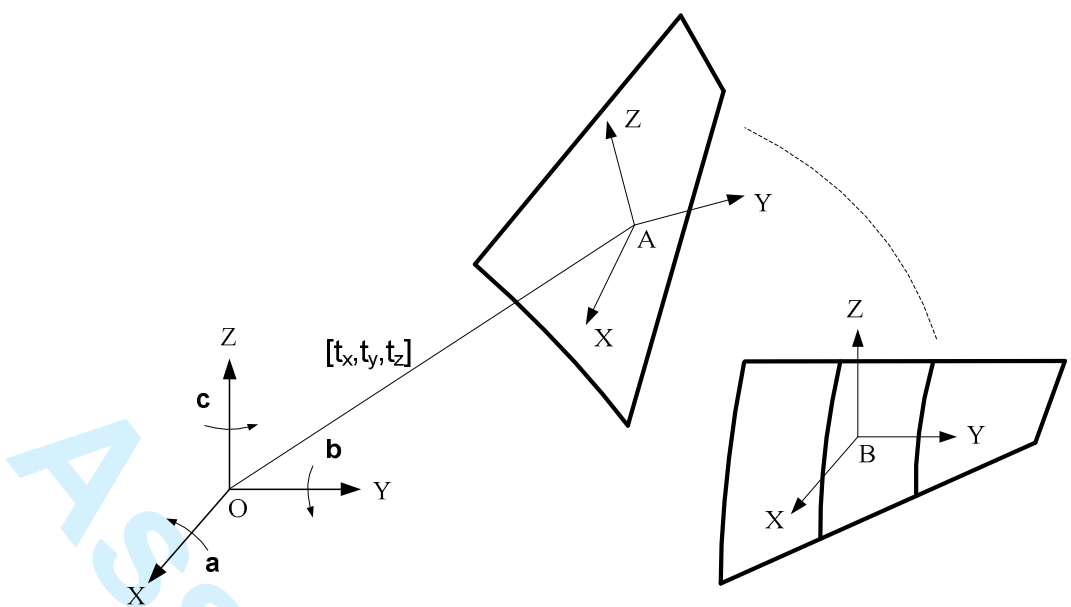

ACS:

Figure 3 Posture transformation

An assembly coordinate system (ACS) is a global reference frame fixed in the assembly space, which is expressed by $\{O-x y z\}$ in figure 3. ACS is taken as the location datum of all components and devices in the automated assembly system.

\section{Posture:}

Attaching a local coordinate system(LCS) $\{A-x y z\}$ with component $\mathrm{A}$, the origin position $\left(t_{x}, t_{y}, t_{z}\right)$ and axis angles a, b, c can be used to describe A's position and orientation in the ACS (i.e. absolute posture). Combining the angles and position together, the absolute posture is expressed as a 6-tuple:

$$
P=\left(a, b, c, t_{x}, t_{y}, t_{z}\right)
$$

With the rotations, $\alpha, \beta, \gamma$, and translations, $x, y, z$, in the ACS, $\{A-x y z\}$ is aligned with the LCS $\{B-x y z\}$ of component $\mathrm{B}$. The relative position and orientation of $\mathrm{B}$ to $\mathrm{A}$ (prior to movement) are defined as relative posture, which is expressed as the 6-tuple:

$$
P_{r}=(\alpha, \beta, \gamma, x, y, z)
$$

The optimal posture calculated in this paper is the relative posture of the panel to the understructure.

\section{Transformation :}

Aligning component A with B, a point $\mathbf{d}$ on A changes its position in the assembly space. The new position can be obtained by a transformation function $A$ :

$$
A(\mathbf{d})=f(\mathbf{r}, \mathbf{t} ; \mathbf{d})=\mathbf{r} \cdot \mathbf{d}+\mathbf{t}
$$

where $\mathbf{r}=\mathbf{r}(\alpha, \beta, \gamma) \in R^{3 \times 3}\left(\mathbf{r}^{T} \mathbf{r}=\mathbf{I},|\mathbf{r}|=1\right)$ is the rotation matrix, $\mathbf{t}=[\mathrm{x}, \mathrm{y}, \mathrm{z}]^{T} \in R^{3 \times 1}$ is the translation vector, and $\mathbf{d}=\left[d_{x}, d_{y}, d_{z}\right]^{T}$ is the coordinate measurement vector in the ACS. The rotation matrix is given by

$$
\mathbf{r}=\left[\begin{array}{ccc}
\cos \gamma & -\sin \gamma & 0 \\
\sin \gamma & \cos \gamma & 0 \\
0 & 0 & 1
\end{array}\right] \cdot\left[\begin{array}{ccc}
\cos \beta & 0 & \sin \beta \\
0 & 1 & 0 \\
-\sin \beta & 0 & \cos \beta
\end{array}\right] \cdot\left[\begin{array}{ccc}
1 & 0 & 0 \\
0 & \cos \alpha & -\sin \alpha \\
0 & \sin \alpha & \cos \alpha
\end{array}\right] .
$$

According to the definition of equation(2), the relative posture of component B to A is ${ }_{A}^{B} P_{r}=h(\mathbf{r}, \mathbf{t})$, where $h$ is a set of formulas as follows:

$$
\left\{\begin{array}{l}
\gamma=\operatorname{atan}\left(\mathbf{r}_{21}, \mathbf{r}_{11}\right) \\
\beta=\operatorname{atan}\left(-\mathbf{r}_{31}, \mathbf{r}_{11} \cos \gamma+\mathbf{r}_{21} \sin \gamma\right) \\
\alpha=\operatorname{atan}\left(\mathbf{r}_{13} \sin \gamma-\mathbf{r}_{23} \cos \gamma, \mathbf{r}_{22} \cos \gamma-\mathbf{r}_{12} \sin \gamma\right) \\
(x, y, z)=\mathbf{t}
\end{array}\right.
$$

where $\mathbf{r}_{m n}$ is the element of the matrix $\mathbf{r}$ in row $\mathrm{m}$, column $\mathrm{n}$.

Inverse transformation: 
When $\mathrm{A}$ is aligned with $\mathrm{B}$, there exists a point $\mathbf{d}_{1}=A(\mathbf{d})=\mathbf{r} \cdot \mathbf{d}+\mathbf{t}$ on $\mathrm{B}$, which can be equally expressed as $\mathbf{d}=\mathbf{r}^{-1} \cdot \mathbf{d}_{1}-\mathbf{r}^{-1} \cdot \mathbf{t}$. Accordingly, an inverse transformation $A^{-1}$ is defined:

$$
A^{-1}(\mathbf{d})=f^{-1}(\mathbf{r}, \mathbf{t} ; \mathbf{d})=\mathbf{r}^{-1} \cdot \mathbf{d}-\mathbf{r}^{-1} \cdot \mathbf{t}=\mathbf{r}^{T} \cdot \mathbf{d}-\mathbf{r}^{T} \cdot \mathbf{t}
$$

The relative posture of component $\mathrm{A}$ to $\mathrm{B}$ is:

\section{Small posture transformation:}

$$
{ }_{B}^{A} P_{r}=h\left(\mathbf{r}^{T},-\mathbf{r}^{T} \cdot \mathbf{t}\right)
$$

By two sequential transformations $A_{1}$ and $A_{2}$, component $\mathrm{A}$ is aligned with $\mathrm{B}$. For any point $\mathbf{d}$ on $\mathrm{A}$, its coordinate is $A_{2}\left(A_{1}(\mathbf{d})\right)$ in the ACS. Thus, an overall transformation can be obtained:

$$
A_{3}=f\left(\mathbf{r}_{3}, \mathbf{t}_{3} ; \mathbf{d}\right)=A_{2}\left(A_{1}\right)=\mathbf{r}_{2}\left(\mathbf{r}_{1} \cdot \mathbf{d}+\mathbf{t}_{1}\right)+\mathbf{t}_{2}
$$

where $\mathbf{r}_{3}=\mathbf{r}_{2} \mathbf{r}_{1}, \mathbf{t}_{3}=\mathbf{r}_{2} \mathbf{t}_{1}+\mathbf{t}_{2}$.

If the motion of transformation $A_{2}$ is differential, i.e. the angles and translations are very small, the equation (4) can be linearized. Expanding to first order terms, the rotation parameters $\mathbf{r}_{2}$ of transformation $A_{2}$ is:

$$
\mathbf{r}_{2}=\left[\begin{array}{ccc}
1 & -\partial \gamma & \partial \beta \\
\partial \gamma & 1 & -\partial \alpha \\
-\partial \beta & \partial \alpha & 1
\end{array}\right]=\mathbf{I}^{3 \times 3}+\left[\begin{array}{ccc}
0 & -\partial \gamma & \partial \beta \\
\partial \gamma & 0 & -\partial \alpha \\
-\partial \beta & \partial \alpha & 0
\end{array}\right]
$$

Further, matrix $\partial \boldsymbol{\theta}$ and vector $\boldsymbol{\partial \theta}_{v}$ are defined as:

$$
\partial \boldsymbol{\theta}=\left[\begin{array}{ccc}
0 & -\partial \gamma & \partial \beta \\
\partial \gamma & 0 & -\partial \alpha \\
-\partial \beta & \partial \alpha & 0
\end{array}\right], \partial \boldsymbol{\theta}_{v}=[\partial \alpha, \partial \beta, \partial \gamma]^{T}
$$

For any vector $\mathbf{x}_{3 \times 1}$, it follows that $\partial \boldsymbol{\theta} \cdot \mathbf{x}=\partial \boldsymbol{\theta}_{v} \times \mathbf{x}$.

Substituting (9) into (8), the overall transformation is:

$$
\mathbf{r}_{3}=\mathbf{r}_{2} \mathbf{r}_{1}=(\mathbf{I}+\partial \boldsymbol{\theta}) \cdot \mathbf{r}_{1}, \quad \mathbf{t}_{3}=(\mathbf{I}+\partial \boldsymbol{\theta}) \cdot \mathbf{t}_{1}+\partial \mathbf{t}
$$

where $\partial \mathbf{t}=[\partial x, \partial y, \partial z]^{T}$.

According to equation(10), the delta transformation in the ACS corresponding to a differential motion are $\Delta \mathbf{r}=\mathbf{r}_{3}-\mathbf{r}_{1}=\partial \boldsymbol{\theta} \cdot \mathbf{r}_{1}, \Delta \mathbf{t}=\mathbf{t}_{3}-\mathbf{t}_{1}=\partial \boldsymbol{\theta} \cdot \mathbf{t}_{1}+\partial \mathbf{t}$. Thus, the small posture transformation (SPT) in the ACS is defined as:

$$
\partial A(\mathbf{d})=f(\mathbf{r}, \mathbf{t}, \partial \boldsymbol{\theta}, \partial \mathbf{t} ; \mathbf{d})=\partial \boldsymbol{\theta} \cdot \mathbf{r} \cdot \mathbf{d}+\partial \boldsymbol{\theta} \cdot \mathbf{t}+\partial \mathbf{t},
$$

where $\mathbf{r}, \mathbf{t}$ are the initial transformation parameters. Especially, if only a SPT is applied, i.e. $\mathbf{r}=\left[\begin{array}{lllllll}1 & 0 & 0 ; 0 & 1 & 0 ; 0 & 0 & 1\end{array}\right]$, $\mathbf{t}=\left[\begin{array}{lll}0 & 0 & 0\end{array}\right]^{T}$, then $\partial A(\mathbf{d})=f(\partial \boldsymbol{\theta}, \partial \mathbf{t} ; \mathbf{d})=\partial \boldsymbol{\theta} \cdot \mathbf{d}+\partial \mathbf{t} \quad$ is obtained.

Combining the initial transformation and SPT, the overall transformation is obtained as $(A+\partial A)(\mathbf{d})$.

\section{Initial posture estimation}

In preliminary alignment, the auxiliary locating points (ALPs) are selected from the wing panel and understructure, as shown in figure 2. By measuring and matching the corresponding ALPs, the initial transformation matrix and posture parameters can be estimated.

Let $\mathbf{d}_{i}, \mathbf{m}_{i}$ be the measurement coordinates of the ALPs of the panel and the understructure respectively. With an initial transformation $A$ applied to the panel, $\mathbf{d}_{i}$ changes to $A\left(\mathbf{d}_{i}\right)$ and the position deviations of the corresponding ALPs are $\delta_{i}=A\left(\mathbf{d}_{i}\right)-\mathbf{m}_{i}$. Clearly, when $\delta_{i}$ becomes smaller in magnitude, the alignment is better. Thus, a posture estimation model is established based on minimizing the position deviations of the ALPs:

$$
\min \sum_{i}^{N} \omega_{i}\left\|A\left(\mathbf{d}_{i}\right)-\mathbf{m}_{i}\right\|
$$

where $N(>3)$ is the number of ALPs; $\omega_{i}$ is a weight parameter on the $i$ th ALP.

Since the panel needs to be transformed by the SPT, $\partial A$, in the refined alignment, the position deviations can be described as $\delta_{i}=A\left(\mathbf{d}_{i}\right)+\partial A\left(\mathbf{d}_{i}\right)-\mathbf{m}_{i}$. The objective of (12) can therefore be modified as: 


$$
\min \sum_{i}^{N} \omega_{i}\left\|A\left(\mathbf{d}_{i}\right)+\partial A\left(\mathbf{d}_{i}\right)-\mathbf{m}_{i}\right\|
$$

When the original transformation, $A$, is known, The objective of (13) is convenient for iterative optimization.

\section{Gap calculation and redistribution}

After the preliminary alignment, the panel is positioned near the understructure, and the assembly gaps between their interfaces are determined. In order to judge whether the gaps are within tolerance, the assembly gaps should be calculated firstly.

\subsection{Gap definition}

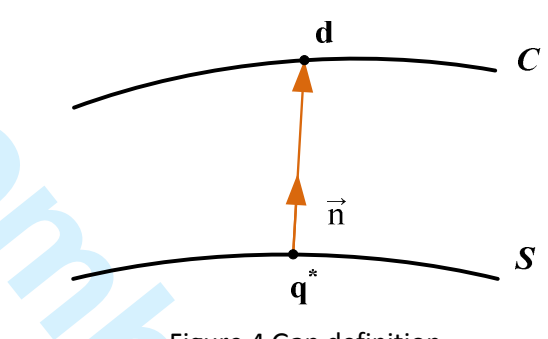

Figure 4 Gap definition

Having two surfaces, $C$ and $S$, for the point $\mathbf{d}$ on surface $C$, the closest point $\mathbf{q}^{*}$ can be found on the surface $S$. The length of $\left|\mathbf{d q}^{*}\right|$ is the distance from surface $C$ to surface $S$ at point $\mathbf{d}$. The nearest point $\mathbf{q}^{*}$ on surface $S$ can be formulated as

$$
\mathbf{q}^{*}=\arg \min \|\mathbf{d}-\mathbf{q}\|, \mathbf{q} \in S
$$

In computational geometry, the point $\mathbf{q}^{*}$ has the following property: the unit normal vector $\mathbf{n}\left(\mathbf{q}^{*}\right)$ is parallel to the vector $\mathbf{d}-\mathbf{q}^{*}$, which can be expressed as

$$
\mathbf{d}-\mathbf{q}^{*}=\varepsilon \cdot \mathbf{n}\left(\mathbf{q}^{*}\right)
$$

where $\varepsilon$ indicates the distance value and direction. The condition $\varepsilon>0$ means that $\mathbf{d}$ is outside of the surface $S$, while $\varepsilon<0$ means that it is inside of the surface $S$, and especially $\varepsilon=0$ denotes that it is on the surface $S$ and the distance $\left|\mathbf{d q}^{*}\right|$ is zero. In the wing panel assembly, the direction of the normal vector is from inner to outer. Thus, the value of assembly gap at point $\mathbf{d}$ is defined as

$$
v=\mathbf{n}\left(\mathbf{q}^{*}\right) \cdot\left(\mathbf{d}-\mathbf{q}^{*}\right)
$$

According to equation(15), the actual mating situation can be inferred, i.e. a positive gap implies non-contact status and the assembly structure is non-integral; while negative gap denotes interference status and the assembly is non-feasible. Given that the gap tolerance range is $T=\left[V^{\mathrm{d}}, V^{\mathrm{u}}\right]$, we can judge whether the gap is out of tolerance by

$$
F=\left\{\begin{array}{l}
0, \text { if } V^{d} \leq v \leq V^{u} \\
1, \text { otherwise }
\end{array}\right.
$$

where $F$ is the out-of-tolerance flag, 0 represents that the gap is in tolerance range while 1 represents out.

\subsection{Gap after transformation}

With the initial transformation $A$, the measurement point $\mathbf{d}$ on the panel changes to $A(\mathbf{d})$. The mating gap at the point is

$$
v_{0}=\mathbf{n}\left(\mathbf{q}^{*}\right) \cdot\left(A(\mathbf{d})-\mathbf{q}^{*}\right)
$$

where $\mathbf{q}^{*}$ is the measurement point of the understructure and $\mathbf{q}^{*}$ is obtained according to (14).

With a sequential SPT, $\partial A$, the coordinate of $\mathbf{d}$ changes to $(A+\partial A)(\mathbf{d})$, and the new gap is updated: 


$$
v_{1}=\mathbf{n}\left(\mathbf{q}_{\text {new }}^{*}\right) \cdot\left\{(A+\partial A)(\mathbf{d})-\mathbf{q}_{\text {new }}^{*}\right\}
$$

where $\mathbf{q}_{\text {new }}^{*}$ is the new closest point on the understructure.

According to equation (17), once the SPT $\partial A$ is changed, the new closest point $\mathbf{q}_{\text {new }}^{*}$ should be re-searched. For an iterative optimization, the closest point searching will be very time-consuming. In particular, if the scanned wing surface is of high density, it is difficult to calculate the new gap to solve an optimal SPT efficiently.

Based on the above considerations, we use a difference method to obtain the delta gap caused by $\partial A$, and add it with the original gap to update the new one indirectly.

Letting $\mathbf{q}_{\text {new }}^{*}=\mathbf{q}^{*}+\delta \mathbf{q}, \quad \mathbf{n}\left(\mathbf{q}_{\text {new }}^{*}\right)=\mathbf{n}\left(\mathbf{q}^{*}\right)+\delta \mathbf{n}$, equation (17) can be expressed as

$$
v_{1}=\left\{\mathbf{n}\left(\mathbf{q}^{*}\right)+\delta \mathbf{n}\right\} \cdot\left\{(A+\partial A)(\mathbf{d})-\left(\mathbf{q}^{*}+\delta \mathbf{q}\right)\right\}
$$

Substituting (11) into (18) yields

$$
v_{1}=\left\{\mathbf{n}\left(\mathbf{q}^{*}\right)+\delta \mathbf{n}\right\} \cdot\left\{(\mathbf{r}+\partial \boldsymbol{\theta} \cdot \mathbf{r}) \cdot \mathbf{d}+\mathbf{t}+\partial \boldsymbol{\theta} \cdot \mathbf{t}+\partial \mathbf{t}-\left(\mathbf{q}^{*}+\delta \mathbf{q}\right)\right\}
$$

The delta gap caused by the SPT is

$$
v_{1}-v_{0}=\left\{\mathbf{n}\left(\mathbf{q}^{*}\right)+\delta \mathbf{n}\right\} \cdot\left\{(\mathbf{I}+\partial \boldsymbol{\theta}) \cdot(\mathbf{r} \cdot \mathbf{d}+\mathbf{t})+\partial \mathbf{t}-\left(\mathbf{q}^{*}+\delta \mathbf{q}\right)\right\}-\mathbf{n}\left(\mathbf{q}^{*}\right) \cdot\left\{\mathbf{r} \cdot \mathbf{d}+\mathbf{t}-\mathbf{q}^{*}\right\}
$$

Expanding the above expression to first order, yields the delta gap $\delta v$

$$
\begin{gathered}
\delta v=v_{1}-v_{0} \approx\left[\mathbf{r} \cdot \mathbf{d}+\mathbf{t}-\mathbf{q}^{*}\right] \cdot \delta \mathbf{n}+\partial \mathbf{t} \cdot \mathbf{n}\left(\mathbf{q}^{*}\right)-\delta \mathbf{q} \cdot \mathbf{n}\left(\mathbf{q}^{*}\right)+\partial \boldsymbol{\theta} \cdot(\mathbf{r} \cdot \mathbf{d}+\mathbf{t}) \cdot \mathbf{n}\left(\mathbf{q}^{*}\right) \\
=\varepsilon \mathbf{n} \cdot \delta \mathbf{n}+\partial \mathbf{t} \cdot \mathbf{n}\left(\mathbf{q}^{*}\right)-\delta \mathbf{q} \cdot \mathbf{n}\left(\mathbf{q}^{*}\right)+\partial \mathbf{\theta} \cdot(\mathbf{r} \cdot \mathbf{d}+\mathbf{t}) \cdot \mathbf{n}\left(\mathbf{q}^{*}\right) \\
v \\
v
\end{gathered}
$$

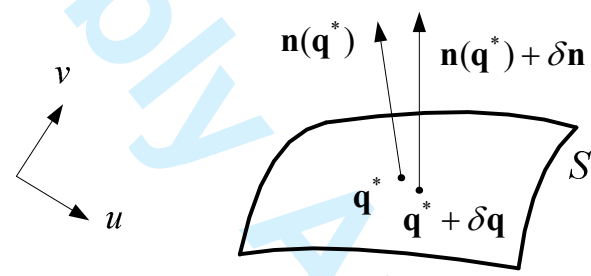

Figure 5 Parametric surface and its geometries

Considering the parametric surface $S(u, v)$ in figure 5, the following mathematical relationships apply

$$
\begin{gathered}
\mathbf{n}\left(\mathbf{q}^{*}\right)=\frac{\partial S\left(u^{*}, v^{*}\right)}{\partial u} \times \frac{\partial S\left(u^{*}, v^{*}\right)}{\partial v} /\left\|\frac{\partial S\left(u^{*}, v^{*}\right)}{\partial u} \times \frac{\partial S\left(u^{*}, v^{*}\right)}{\partial v}\right\| \\
\delta \mathbf{q}=\frac{\partial S\left(u^{*}, v^{*}\right)}{\partial u} \Delta u+\frac{\partial S\left(u^{*}, v^{*}\right)}{\partial v} \Delta v \\
\delta \mathbf{n}\left(\mathbf{q}^{*}\right)=\frac{\partial \mathbf{n}\left(u^{*}, v^{*}\right)}{\partial u} \Delta u+\frac{\partial \mathbf{n}\left(u^{*}, v^{*}\right)}{\partial v} \Delta v
\end{gathered}
$$

According to the above definitions, $\mathbf{n}\left(\mathbf{q}^{*}\right) \cdot \delta \mathbf{n}\left(\mathbf{q}^{*}\right)=0$ and $\delta \mathbf{q} \cdot \mathbf{n}\left(\mathbf{q}^{*}\right)=0$ can be obtained. Substituting the expressions into equation (20) yields

$$
\delta v \approx \partial \mathbf{t} \cdot \mathbf{n}\left(\mathbf{q}^{*}\right)+\partial \boldsymbol{\theta} \cdot(\mathbf{r} \cdot \mathbf{d}+\mathbf{t}) \cdot \mathbf{n}\left(\mathbf{q}^{*}\right)
$$

The matrix $\partial \boldsymbol{\theta}$ in (21) is skew-symmetric, and according to (9), $\partial \boldsymbol{\theta} \cdot \mathbf{b}=\partial \boldsymbol{\theta}_{v} \times \mathbf{b}$. Also, for any vectors, $\mathbf{u} \times \mathbf{v} \cdot \mathbf{w}=\mathbf{u} \cdot(\mathbf{v} \times \mathbf{w})$ (see proof in the appendix). Substituting the equations into equation (21) yields

$$
\begin{aligned}
\delta v & \approx \partial \mathbf{t} \cdot \mathbf{n}\left(\mathbf{q}^{*}\right)+\partial \boldsymbol{\theta}_{v} \times(\mathbf{r} \cdot \mathbf{d}+\mathbf{t}) \cdot \mathbf{n}\left(\mathbf{q}^{*}\right) \\
& =\partial \mathbf{t} \cdot \mathbf{n}\left(\mathbf{q}^{*}\right)+\partial \boldsymbol{\theta}_{v} \cdot\left\{\left[\mathbf{r} \cdot \mathbf{d}+\mathbf{t}-\varepsilon \mathbf{n}\left(\mathbf{q}^{*}\right)\right] \times \mathbf{n}\left(\mathbf{q}^{*}\right)\right\} \\
& =\partial \mathbf{t} \cdot \mathbf{n}\left(\mathbf{q}^{*}\right)+\partial \boldsymbol{\theta}_{v} \cdot\left\{\mathbf{q}^{*} \times \mathbf{n}\left(\mathbf{q}^{*}\right)\right\}
\end{aligned}
$$

where $\left\{\mathbf{n}\left(\mathbf{q}^{*}\right), \mathbf{q}^{*} \times \mathbf{n}\left(\mathbf{q}^{*}\right)\right\}$ is the Plöcker coordinate of the normal line of surface $S$ at point $\mathbf{q}^{*}$ (Helmut et al., 1999).

According to equation (16) and (22), the relationship of the gap and the transformations is obtained as 


$$
v=v_{0}+\delta v=\mathbf{n}\left(\mathbf{q}^{*}\right) \cdot\left[A(\mathbf{d})-\mathbf{q}^{*}\right]+\partial \mathbf{t} \cdot \mathbf{n}\left(\mathbf{q}^{*}\right)+\partial \boldsymbol{\theta}_{v} \cdot\left[\mathbf{q}^{*} \times \mathbf{n}\left(\mathbf{q}^{*}\right)\right],
$$

where $A$ is the initial transformation, $\left(\partial \boldsymbol{\theta}_{v}, \partial \mathbf{t}\right)$ are the SPT parameters. Analogous to equation (13), with a known $A$, equation (23) degenerates into a linear function with respect to SPT minims. When the SPT parameters are solved, the overall transformation is obtained according to equation (10).

\subsection{Gap redistribution}

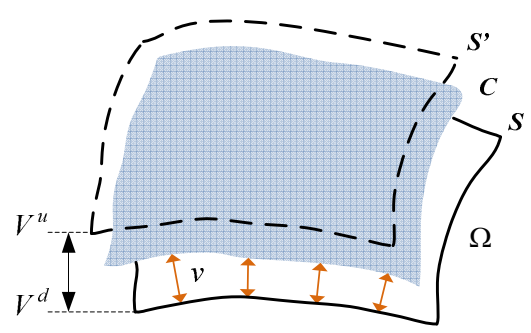

Figure 6 Mating region

According to equation (23), the assembly gap can be changed by adjusting the SPT parameters. Considering the mating region $\Omega$ in figure $6, V^{u}, V^{d}$ are the up and down gap tolerances, $v$ is the gap at any point in the region. The assembly requirements that gaps should be within tolerance can be expressed as $V^{d} \leq v \leq V^{u}$. When the gaps are within tolerance, the overall gap is required to be as small as possible, so that fewer fillers are required in processes, such as shimming, sealing, etc. To meet the requirements, a gap minimization model with tolerance constraints is established:

$$
\min \sum_{k=1}^{M}\left\|v_{k}\right\| \text { s.t. } V^{d} \leq v_{k} \leq V^{u}
$$

where $M$ is the number of the points in $\Omega$.

Objective (24) can be used to optimize the gaps in region $\Omega$. However, with the optimization, the gaps of other regions will be increased or decreased. Thus, modifying formula (24) into a weighted model with multi-region gap constraints yields:

$$
\min \sum_{j=1}^{R} \sum_{k=1}^{M} \omega_{j}\left\|v_{j k}\right\| \text { s.t. } V_{j}^{d} \leq v_{j k} \leq V_{j}^{u}
$$

where $\omega_{j}, 1 \leq j \leq R$ is the gap weight, which indicates the gap importance; $V_{j}^{u}, V_{j}^{d}$ are the gap tolerance of region $j$; $v_{j k}$ is the gap value of point $k$ in region $j ; M$ is the number of the points in a local region, and $R$ is the number of mating regions.

Letting $\varepsilon_{j}=V_{j}^{u}-V_{j}^{d}$, the weight $\omega_{j}$ is defined as:

$$
\omega_{j}=\frac{1 / \varepsilon_{j}}{\sum_{1}^{R} 1 / \varepsilon_{j}}
$$

In the wing assembly, $\varepsilon_{j} \leq 1$, and a gap of smaller tolerance will get a larger weight.

\section{Optimal posture evaluation model}

Objectives (13) and (25) are used in preliminary and refined alignments, respectively. In order to combine the two alignments together and establish a unified evaluation model, a comprehensive error function $E\left(\mathbf{r}, \mathbf{t}, \partial \boldsymbol{\theta}_{v}, \partial \mathbf{t}\right)$ is defined as: 


$$
\begin{aligned}
& E\left(\mathbf{r}, \mathbf{t}, \partial \boldsymbol{\theta}_{v}, \partial \mathbf{t}\right) \\
& =\sum_{i}^{N} \omega_{i}\left\|\delta_{i}\right\|+\sum_{j=1}^{R} \sum_{k=1}^{M} \omega_{j}\left\|v_{j k}\right\| \\
& =\sum_{i}^{N} \omega_{i}\left\|(A+\partial A)\left(\mathbf{d}_{i}\right)-\mathbf{m}_{i}\right\|+ \\
& \sum_{j=1}^{R} \sum_{k=1}^{M} \omega_{j}\left\|\left\{\mathbf{n}\left(\mathbf{q}^{*}\right) \cdot\left[A\left(\mathbf{d}_{k}\right)-\mathbf{q}^{*}\right]+\partial \mathbf{t} \cdot \mathbf{n}\left(\mathbf{q}^{*}\right)+\partial \boldsymbol{\theta}_{v} \cdot\left[\mathbf{q}^{*} \times \mathbf{n}\left(\mathbf{q}^{*}\right)\right]\right\}_{j}\right\|
\end{aligned}
$$

where $\mathbf{r}, \mathbf{t}$ are the initial transformation parameters, and $\partial \boldsymbol{\theta}_{v}, \partial \mathbf{t}$ are the SPT parameters.

Taking the above error function as a minimization objective and gap tolerances as constraints, an optimal posture evaluation model is established as:

$$
\left\{\begin{array}{l}
\min \left\{\sum_{i}^{N} \omega_{i}\left\|\delta_{i}\right\|+\sum_{j=1}^{R} \sum_{k=1}^{M} \omega_{j}\left\|v_{j k}\right\|\right\} \\
\text { s.t. } C_{k}^{1}=V_{j k}^{u}-v_{j k} \geq 0, C_{k}^{2}=v_{j k}-V_{j k}^{d} \geq 0
\end{array}\right.
$$

where $C_{k}$ is the constraint condition at point $k ; V_{j k}^{u}, V_{j k}^{d}$ are the gap tolerances at point $k$ in region $j$, and the points in the same region have the same tolerances.

By solving the constrained objective (28), the best transformation parameters $\mathbf{r}, \mathbf{t}, \partial \boldsymbol{\theta}_{v}, \partial \mathbf{t}$ of the panel are obtained, with the known parameters the optimal posture of the panel relative to the understructure can be calculated according to (5), (7) and (10).

\section{Optimization algorithm}

\subsection{Main program}

For a constrained optimization problem such as $\min f(x)$, s.t. $c_{i}(x) \geq 0$, the Lagrange method is widely used for its solution. The problem is first modified to $\min f(x)$, s.t. $c_{i}(x)-z_{i}^{2}=0$, then the Lagrangian function is constructed:

$$
L(x, z, \lambda, \sigma)=f(x)-\sum \lambda_{i}\left[c_{i}(x)-z_{i}^{2}\right]+\sigma / 2 \sum\left[c_{i}(x)-z_{i}^{2}\right]^{2} .
$$

where $z_{i}$ is the auxiliary variable, $\lambda_{i}$ is the multiplier, and $\sigma$ is the penalty factor.

Setting the partial derivatives with respect to component $\mathrm{z}$ to zero in equation (29), the auxiliary variable $\mathrm{z}$ is eliminated and a new Lagrangian function is obtained:

$$
L(x, \lambda, \sigma)=f(x)+1 / 2 \sigma \sum\left\{\max \left[0, \lambda_{i}^{2}-\sigma c_{i}(x)\right]^{2}-\lambda_{i}^{2}\right\} .
$$

The iterative formulas and termination condition are:

$$
\begin{aligned}
& \sigma_{n+1}=\mu \sigma_{n} \\
& \left(\lambda_{n+1}\right)_{i}=\max \left\{0,\left(\lambda_{n}\right)_{i}-\sigma c_{i}\left(x_{n}\right)\right\} \\
& \tau_{n}=\left\{\sum \min \left[c_{i}\left(x_{n}\right),\left(\lambda_{n}\right)_{i} / \sigma_{n}\right]^{2}\right\}^{1 / 2}<\varepsilon
\end{aligned}
$$

The PHR algorithm is a well-known augmented Lagrange method (Birgin et al., 2005), and the PHR based optimization method is listed in Algorithm 1 for the solution of variable $x=(\partial \alpha, \partial \beta, \partial \gamma, \partial x, \partial y, \partial z)$. 
Algorithm 1 PHR optimization:

\author{
Input: $\quad E(x), C(x), r, t$ \\ Output: $r^{\prime}, t^{\prime}$ \\ Procedure:
}

a) Initialization:

$$
\begin{aligned}
& \lambda_{1}>0, \sigma_{1}>0, \mu>1, \varepsilon>0 \\
& 0<\omega<1, x_{1}, n=1
\end{aligned}
$$

b) BFGS algorithm:

$$
x_{n+1}=\text { procedure } 1\left(x_{n}\right)
$$

c) Update L-function:

$$
\begin{aligned}
& \text { if } \left.\left(\tau_{n}<\varepsilon\right) \quad x^{*}=x_{n+1} \text {, goto } d\right) \\
& \text { end if } \\
& \text { if }\left(\tau_{n+1} / \tau_{n}>\omega\right) \quad \sigma_{n+1}=\mu \sigma_{n} \\
& \text { end if } \\
& \left(\lambda_{n+1}\right)_{i}=\max \left\{0,\left(\lambda_{n}\right)_{i}-\sigma c_{i}\left(x_{n}\right)\right\} \\
& n=n+1, \text { goto } b) \\
& \text { d) Calculate new r,t: } \\
& r^{\prime}=\left(I_{3 \times 3}+\partial \theta\left(x^{*}\right)\right) \cdot r \\
& t^{\prime}=\left(I_{3 \times 3}+\partial \theta\left(x^{*}\right)\right) \cdot t+\partial t\left(x^{*}\right)
\end{aligned}
$$

The Broyden-Fletcher-Goldfarb-Shanno (BFGS) algorithm is considered as a leading Quasi-Newton method, which has global convergence and super-linear convergence speed (Nocedal, et al.,2006). The algorithm is especially suitable for the minimum optimization problem with mass data. In Procedure 1, the BFGS algorithm is used to minimize the given Lagrangian function $L\left(x_{n}\right)$ in Algorithm 1.

\title{
Procedure 1 BFGS algorithm:
}

a) Initialization:

$$
\begin{aligned}
& \varepsilon>0, \rho>0,0<l<u<1, \\
& H_{1}=I_{6 \times 6}, k=1, x_{1}=x_{n} .
\end{aligned}
$$

b) Search direction and step:

$$
\begin{aligned}
& d_{k}=-H_{k} \cdot \nabla L\left(x_{k}\right) \\
& \lambda_{k}=l \operatorname{search}\left(L\left(x_{k}\right), \rho, l, u, x_{k}, d_{k}\right)
\end{aligned}
$$

c) Calculate new $\mathrm{x}$ :

$$
\begin{aligned}
& x_{k+1}=x_{k}+\lambda_{k} d_{k} \\
& \text { if }\left(\nabla L\left(x_{k+1}\right)<\varepsilon\right) \text { return } x_{k+1} \\
& \text { end if }
\end{aligned}
$$

d) Update matrix $\mathrm{H}$ :

$$
\begin{aligned}
& y_{k}=\nabla L\left(x_{k+1}\right)-\nabla L\left(x_{k}\right) \\
& s_{k}=x_{k+1}-x_{k} \\
& v_{1}=\frac{s_{k} y_{k}^{T}}{y_{k}^{T} s_{k}}, v_{2}=\frac{y_{k} s_{k}^{T}}{y_{k}^{T} s_{k}}, v_{3}=\frac{s_{k} s_{k}^{T}}{y_{k}^{T} s_{k}} \\
& H_{k+1}=\left(I-v_{1}\right) \cdot H_{k} \cdot\left(I-v_{2}\right)+v_{3} \\
& k=k+1, \text { goto } b)
\end{aligned}
$$

The workflow for the solution of objective (28) is shown in figure 7. Firstly, setting $\omega_{i}=1, \omega_{j}=0$, $\partial \mathbf{r}=\mathbf{0}, \partial \mathbf{t}=\mathbf{0}$, the initial transformation $\mathbf{r}, \mathbf{t}$ is solved by SVD algorithm. Secondly, substituting the known $\mathbf{r}, \mathbf{t}$ into equation (28), the comprehensive objective function $E$ and constraints $C$ are obtained. Thirdly, taking the functions $E(x), C_{k}(x)$ as $f(x), c_{i}(x)$ in equation (30), the PHR method is used to solve the SPT $\partial \mathbf{r}, \partial \mathbf{t}$ and the overall transformation $\mathbf{r}^{\prime}, \mathbf{t}^{\prime}$. Lastly, judging whether the stop condition is met, if YES then calculate the optimal 
1

2

3

4

5

7

8

9

10

11

12

13

14

15

16

17

18

19

20

21

22

23

24

25

26

27

28

29

30

31

32

33

34

35

36

37

38

39

40

41

42

43

44

45

46

47

48

49

50

51

52

53

54

55

56

57

58

59

60

posture by (5) and (7), and terminate the algorithm; if NO then let $\mathbf{r}=\mathbf{r}^{\prime}, \mathbf{t}=\mathbf{t}^{\prime}$ and repeat the previous steps until termination.

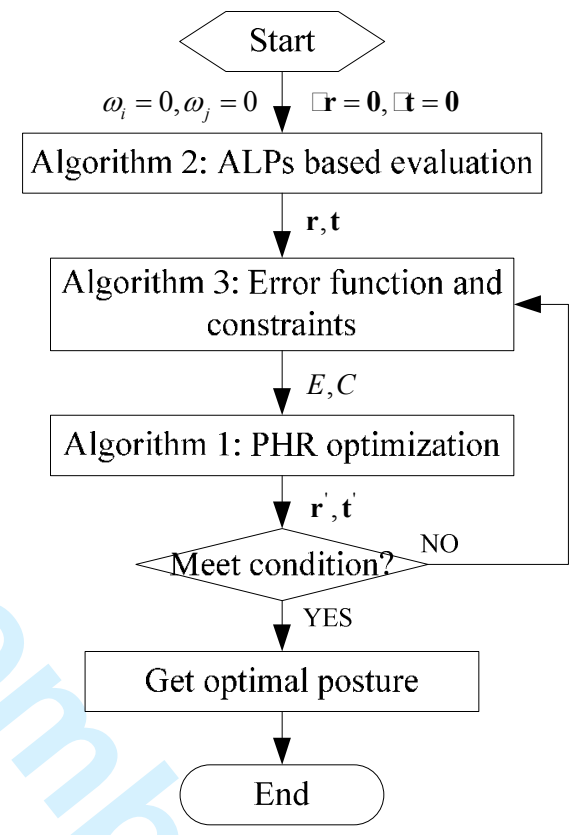

Figure 7 Main program workflow

\subsection{Key algorithms}

The matrix based SVD algorithm can directly (i.e. non-iteratively) solve the transformation parameters with good convergence and precision. Based on the SVD algorithm, Algorithm 2 centralizes the ALPs first and decomposes the correlation matrix to obtain the initial transformation parameters $\mathbf{r}, \mathbf{t}$.

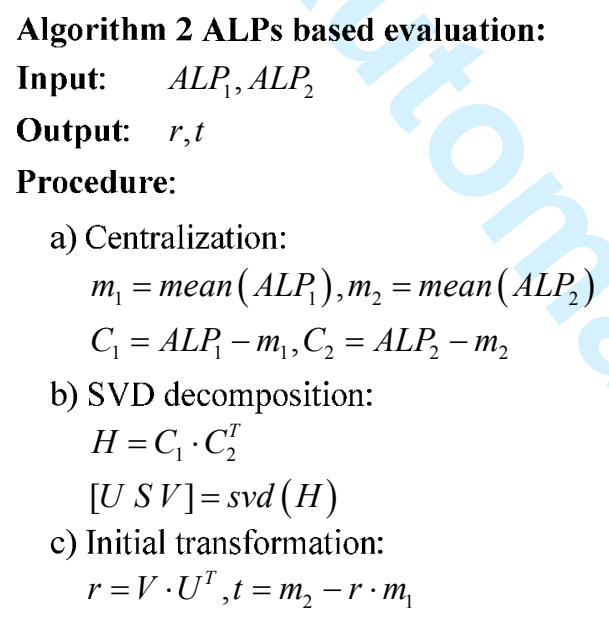

Algorithm 3 is the calculation method of the error function and constraint function. In step b), a micro plane is fitted with the k-neighbor points in $D_{2}$ (k is set 24 30), and the normal vector of the micro plane is took as the one of the surface at the specified point (Philip et al., 2005). All of the KD-tree functions (Matlab and $\mathrm{C}++$ ) involved in Algorithm 3 are available from the KD-tree toolbox (https://github.com/ataiya/kdtree). 


\section{Algorithm 3 Error and constraints calculation:}

Input: $\quad D_{1}, D_{2}, r, t, A L P_{1}, A L P_{2}$

Output: $E, C$

Procedure:

a) Corresponding pairs:

$$
\begin{aligned}
& D_{1}=r \cdot D_{1}+t \\
& t r=k d t r e{ }^{\prime} \text { build }\left(D_{1}\right) \\
& {\left[i d x_{1}, \text { dis } s_{1}\right]=\text { nearest_neighbor }\left(t r, D_{2}\right)} \\
& D_{1}=D_{1}(i d x), u=\left(D_{1}-D_{2}\right) /\left\|D_{1}-D_{2}\right\|
\end{aligned}
$$

b) Gap and constraints:

$$
\begin{aligned}
& \operatorname{tr}=k d t r e{ }_{-} \text {build }\left(D_{2}\right) \\
& \text { for }\left(j=1 ; j \leq \operatorname{size}\left(D_{2}\right) ; j++\right) \\
& \quad i d x_{2}=k_{-} \text {nearest_neighbor }\left(t r, D_{2}(j), k\right) \\
& \quad n_{j}=\text { MicroPlaneFitting }\left(D_{2}\left(i d x_{2}\right)\right) \\
& \quad v(j)=\operatorname{dis} s_{1}(j) \cdot \operatorname{dot}\left(u_{j}, n_{j}\right) \\
& \quad \delta v(j)=n_{j} \cdot \partial t+D_{2}(j) \times n_{j} \cdot \partial \theta_{v} \\
& \quad v(j)=v(j)+\delta v(j) \\
& \quad c(j)=V_{j}^{u}-v(j) \\
& \quad c\left(j+\operatorname{size}\left(D_{2}\right)\right)=v(j)-V_{j}^{d} \\
& \text { end for } \\
& \text { Comprehensive evaluation error: } \\
& \delta=r \cdot A L P_{1}+t-A L P_{2} \\
& E=\sum \omega_{i} \delta_{i}+\sum \omega_{j} v_{j} \\
& C=c
\end{aligned}
$$$$
n_{j}=\text { MicroPlaneFitting }\left(D_{2}\left(i d x_{2}\right)\right)
$$$$
c\left(j+\operatorname{size}\left(D_{2}\right)\right)=v(j)-V_{j}^{d}
$$

c) Comprehensive evaluation error :

\section{Examples}

In the following examples, the parameters are set as $\lambda_{1}=0.1, \sigma_{1}=0.7, \mu=1.5, \varepsilon=0.0001, \omega=0.8$ in Algorithm 1, and $\varepsilon=0.0001, \rho=0.01, l=0.15, u=0.85$ in Procedure 1 .

\subsection{Simulated model data}

The simulated model of the wing is from a spherical crown of radius $R=20 \mathrm{~m}$ and height $h=160 \mathrm{~mm}$ as shown in figure 8. The understructure is composed of \#1 6 frames, and the gap tolerance are $[0,1] \mathrm{mm}$ for $\# 1 \sim 3$ frames, $[0$, $0.5] \mathrm{mm}$ for $\# 4 \sim 5$ frames, $[0,0.6] \mathrm{mm}$ for $\# 6$ frame. The nominal center coordinates of $\# 1 \sim 4$ points are (-1433.881, $-255.754,-3.640) \mathrm{mm},(-238.662,659.786,7.890) \mathrm{mm},(1055.256,613.460,27.613) \mathrm{mm}$ and $(1715.070,-231.862$, 13.497)mm respectively. 


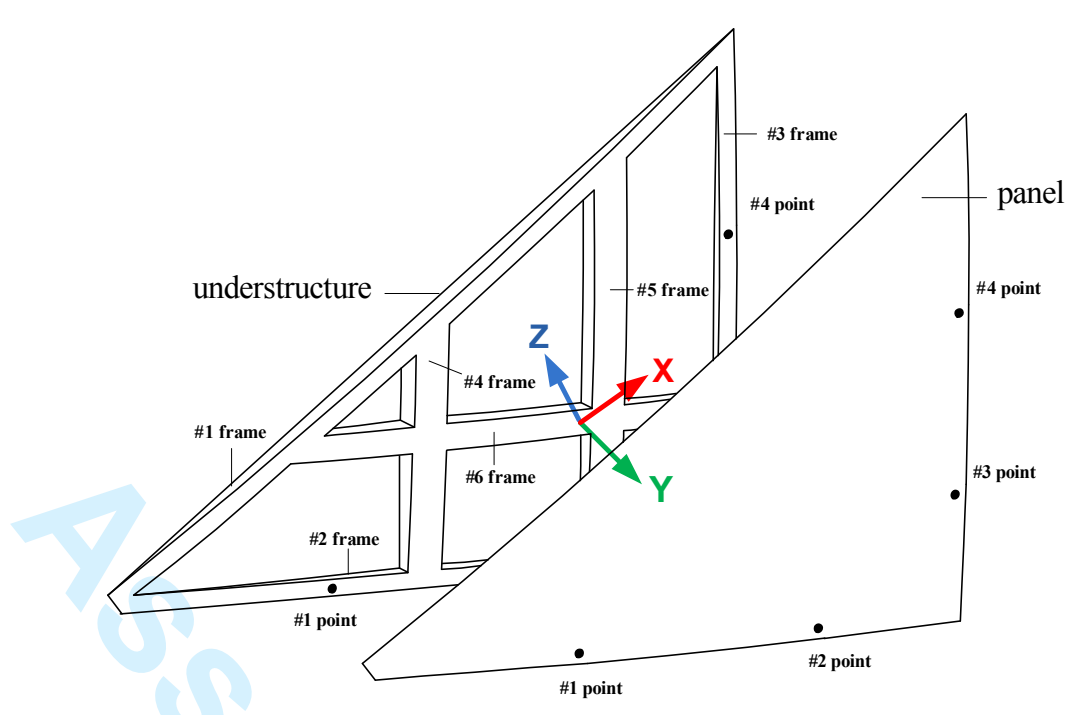

Figure 8 Simulated model of the wing

Rotating the panel by $5^{\circ}, 4^{\circ},-7^{\circ}$ and translating it by $100 \mathrm{~mm}, 100 \mathrm{~mm}$ and $-100 \mathrm{~mm}$ along x-, y-, and z-axes respectively, the panel is separated from the understructure (figure 9). The inner surface of the panel is added with global and regional deformation errors, and the ALPs coordinates are mixed with Gaussian distribution errors $N_{1} \sim(0$, $0.3), \quad N_{2} \sim(0,0.01)$. The actual ALPs coordinates are $(-1352.287,22.047,-25.552) \mathrm{mm},(-51.657,780.211$, -18.071)mm, (1224.814, 575.345, -92.728)mm, (1769.628, -338.698, -226.218)mm of \#1 4 points on the panel, and $(-1433.871,-255.751,-3.632) \mathrm{mm},(-238.654,659.789,7.896) \mathrm{mm},(1055.256,613.460,27.619) \mathrm{mm},(1715.078$, $-231.853,13.499) \mathrm{mm}$ of the corresponding points on the understructure.

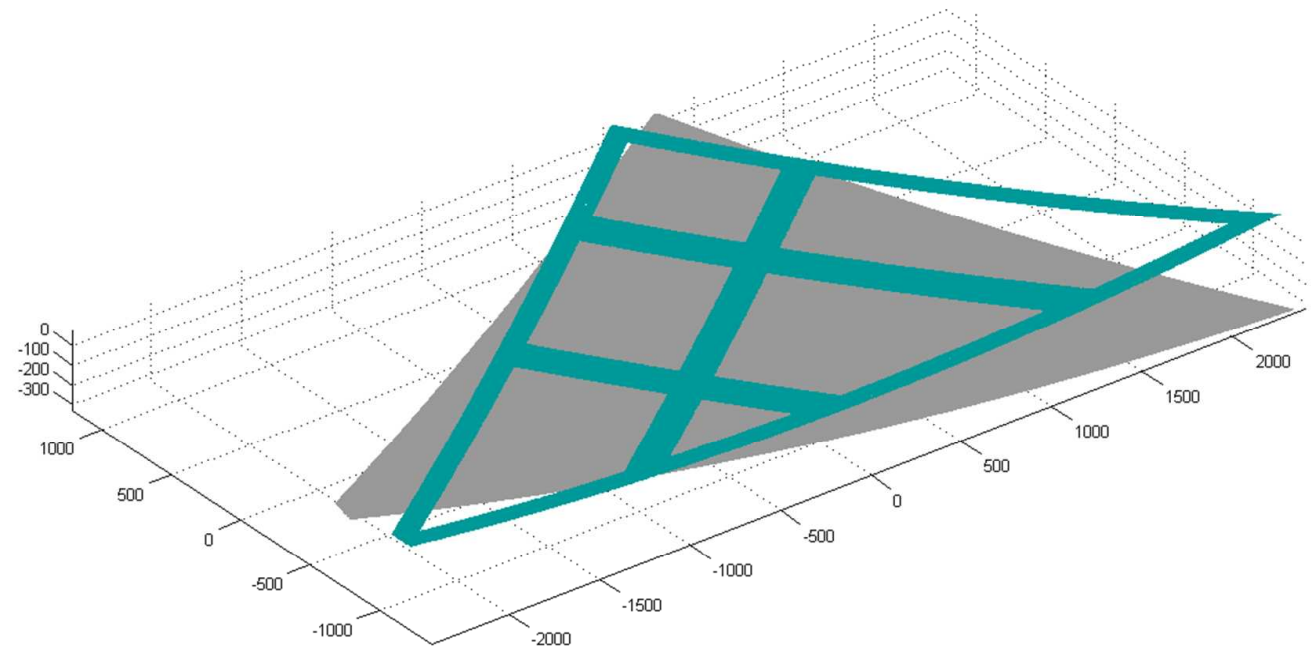

Figure 9 The separated panel and understructure

According to the above actual coordinates of ALPs, the initial transformation is solved as

$$
\mathbf{r}=\left[\begin{array}{l}
0.99013162,-0.12153165,-0.06978129 \\
0.12739319,0.98804687,0.08680062 \\
0.05839816,-0.09483370,0.99377876
\end{array}\right], \quad \mathbf{t}=[-94.00842,-102.99711,102.83392]
$$

The initial posture is (4.9918 deg, $4.0014 \mathrm{deg},-6.9976 \mathrm{deg}, 100.197 \mathrm{~mm}, 100.093 \mathrm{~mm},-99.814 \mathrm{~mm})$. Transforming the panel with the initial parameters, the gaps of the mating surfaces are shown in figure 10 (a). 


\begin{tabular}{cccccccc}
\hline Point & $\mathrm{X} / \mathrm{mm}$ & $\mathrm{Y} / \mathrm{mm}$ & $\mathrm{Z} / \mathrm{mm}$ & $\mathrm{Nx} / \mathrm{mm}$ & $\mathrm{Ny} / \mathrm{mm}$ & $\mathrm{Nz} / \mathrm{mm}$ & $\delta \mathrm{v} / \mathrm{mm}$ \\
\hline A & 88.053 & 382.906 & -64.107 & -0.0038 & 0.0408 & -0.9992 & 0.0377 \\
B & 978.445 & -654.844 & -49.407 & 0.0446 & 0.0307 & -0.9985 & -0.1433 \\
C & 2085.979 & 1136.462 & 30.158 & 0.1015 & 0.0063 & -0.9948 & -0.3124 \\
D & 423.916 & 1035.666 & 53.307 & 0.0204 & 0.1084 & -0.9939 & -0.2006
\end{tabular}
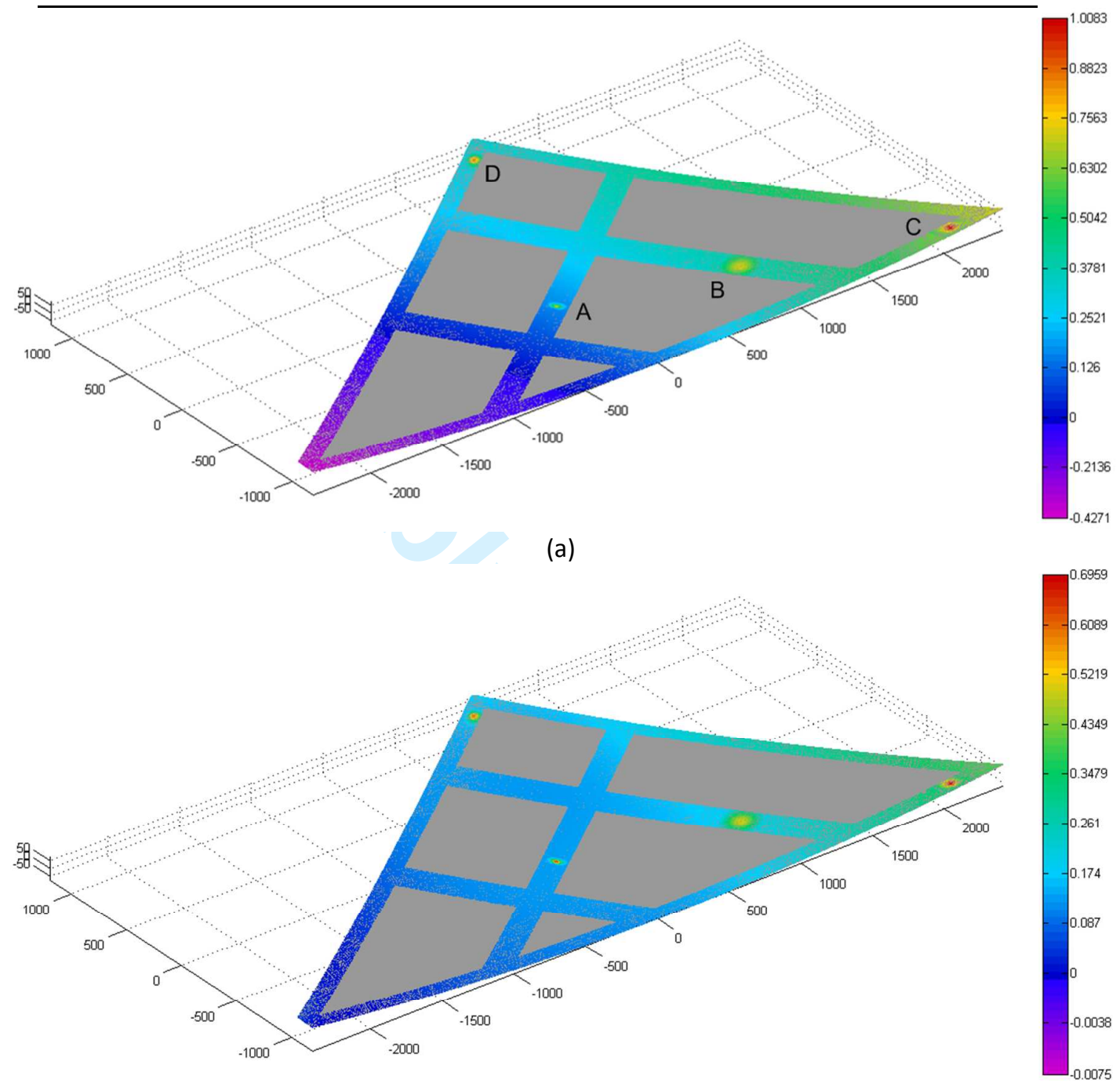

(b)

Figure 10 Gap cloud map

Following the workflow in figure 7 , the SPT variable is solved as $\mathrm{x}=(0.0057 \mathrm{deg},-0.0114 \mathrm{deg}, 0.0057 \mathrm{deg}$, $-0.03 \mathrm{~mm}, 0.05 \mathrm{~mm}, 0.02 \mathrm{~mm})$. The optimal transformation is obtained as:

$$
\mathbf{r}^{\prime}=\left[\begin{array}{l}
0.99010718,-0.12161151,-0.06998871 \\
0.12748636,0.98804420,0.08669424 \\
0.05860892,-0.09475920,0.99377346
\end{array}\right], \mathbf{t}^{\prime}=[-94.04868,-102.96680,102.82482]
$$

The optimal posture is (4.9857 deg, $4.0133 \mathrm{deg},-7.0024 \mathrm{deg}, 100.219 \mathrm{~mm}, 100.042 \mathrm{~mm},-99.840 \mathrm{~mm})$. The updated gap cloud map is revealed in figure 10(b), and the delta gaps at A, B, C, D in figure 10(a) are listed in table 1.

Because the gaps at point A, B, C, D in figure 10(a) are relative large, it is more typical and evidential to choose them for illustrating the control results. The same reason is also considered for the points in table 2 . 
As shown in figure 10, the overall gap between the mating surfaces is optimized, and the gaps at $\mathrm{B}, \mathrm{C}$ are controlled within tolerance by the SPT. In table 1, the delta gaps (positive and negative) are determined by the point coordinates, normal vectors, and SPT variables. Points of larger coordinates will have larger delta gaps.

\subsection{Actual product data}

The wing of a new generation fighter plane is made largely of composite material, and the requirements on the assembly gap are very strict. Because the actual wing is composed of many parts with complex structures and the project is classified, a simplified version of the panel and understructure is given in figure 11. The center coordinates of \#1 4 ALPs on the panel are (1761.354, -128.027, -213.314)mm, (1760.760, 925.763, -201.151)mm, (554.587, $1651.382,-75.975) \mathrm{mm}$ and $(-390.990,1635.491,-85.565) \mathrm{mm}$, and the corresponding coordinates of the ALPs on the understructure are $(1761.216,-128.001,-63.354) \mathrm{mm},(1760.515,925.791,-51.401) \mathrm{mm},(554.256,1651.312$, $73.663) \mathrm{mm}$ and $(-391.320,1635.323,64.132) \mathrm{mm}$. The gap tolerance are $[0,0.4] \mathrm{mm}$ for the fuel tank, $[0,0.5] \mathrm{mm}$ for the wing root, and $[0,1] \mathrm{mm}$ elsewhere.

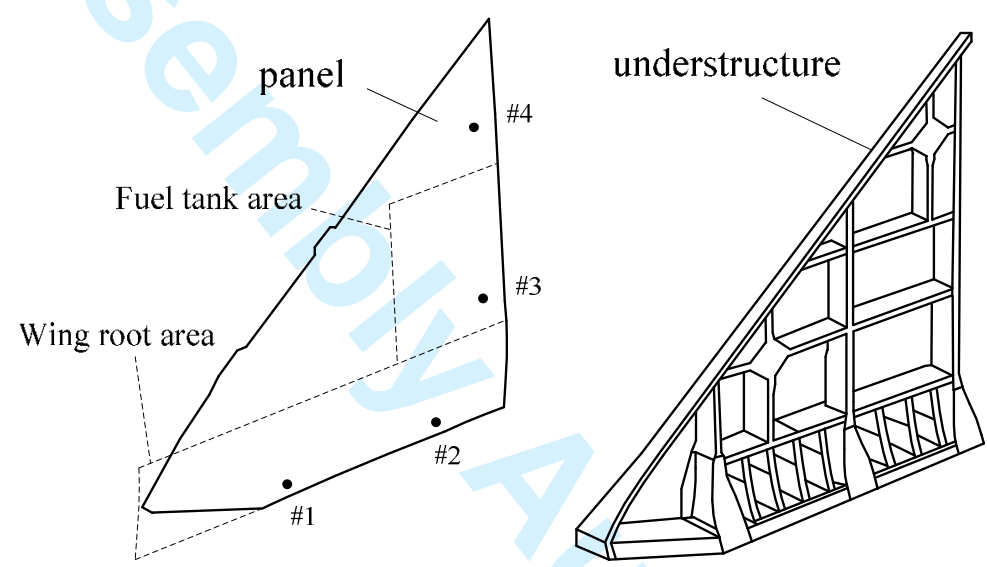

Figure 11 Structures of the wing

According to the workflow and algorithms in figure 7, the initial and optimal postures are $(0.0114 \mathrm{deg},-0.0034$ deg, $-0.0058 \mathrm{deg}, 0.147 \mathrm{~mm}, 0.141 \mathrm{~mm},-150.039 \mathrm{~mm})$, and $(0.0171 \mathrm{deg}, 0.0023 \mathrm{deg},-0.0058 \mathrm{deg}, 0.077 \mathrm{~mm}$, $0.061 \mathrm{~mm},-150.069 \mathrm{~mm})$. The SPT variable is $\mathrm{x}=(-0.0057 \mathrm{deg},-0.0057 \mathrm{deg}, 0 \mathrm{deg}, 0.07 \mathrm{~mm}, 0.08 \mathrm{~mm}, 0.03 \mathrm{~mm})$.

The assembly gaps of the mating surfaces are shown in figure 12 . The delta gaps caused by the SPT at A, B, C, D, E, F in figure 12(a) are listed in table 2.

Table 2 Delta gaps at 6 points

\begin{tabular}{cccccccc}
\hline Point & $\mathrm{X} / \mathrm{mm}$ & $\mathrm{Y} / \mathrm{mm}$ & $\mathrm{Z} / \mathrm{mm}$ & $\mathrm{Nx} / \mathrm{mm}$ & $\mathrm{Ny} / \mathrm{mm}$ & $\mathrm{Nz} / \mathrm{mm}$ & $\delta \mathrm{v} / \mathrm{mm}$ \\
\hline A & -1790.882 & 1805.411 & 22.649 & 0.0569 & 0.0239 & -0.9981 & 0.3348 \\
B & 438.823 & 276.221 & 70.631 & 0.0039 & -0.0072 & -0.9999 & -0.0466 \\
C & 1527.401 & -1622.53 & -17.601 & -0.4559 & -0.0490 & -0.8887 & -0.3431 \\
D & 1522.312 & -664.614 & -2.934 & -0.3191 & 0.0285 & -0.9473 & -0.2557 \\
E & 1440.773 & 97.302 & 32.537 & -0.1864 & -0.0004 & -0.9825 & -0.1739 \\
F & 1443.690 & 862.611 & 23.025 & -0.1946 & -0.0111 & -0.9808 & -0.1005 \\
\hline
\end{tabular}




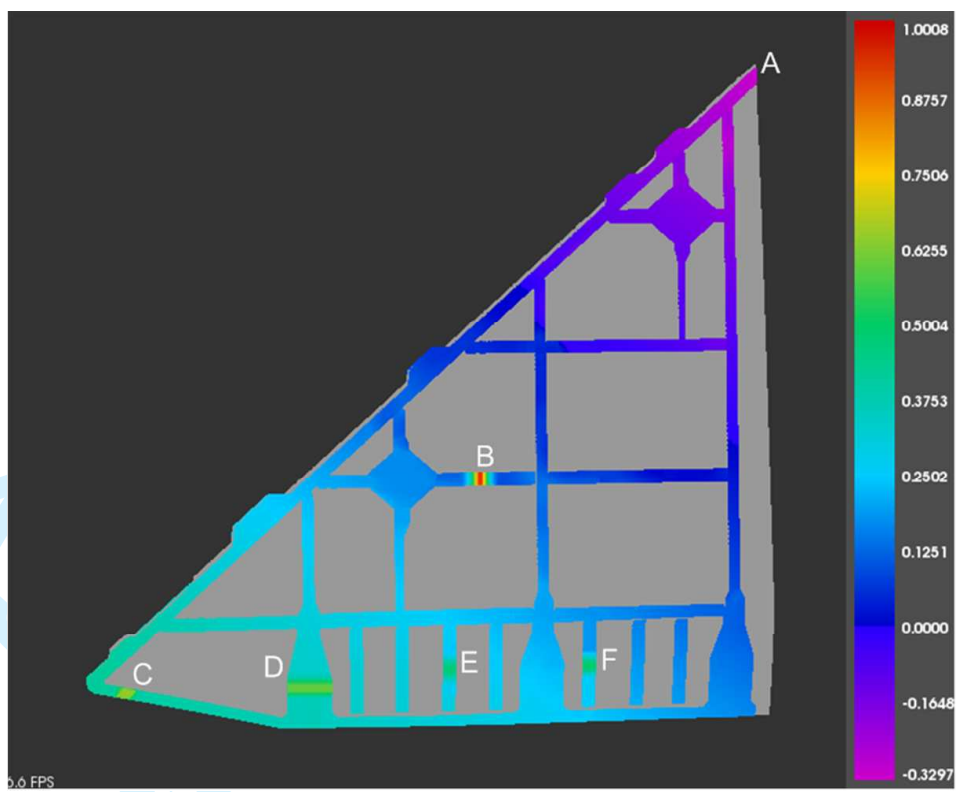

(a)

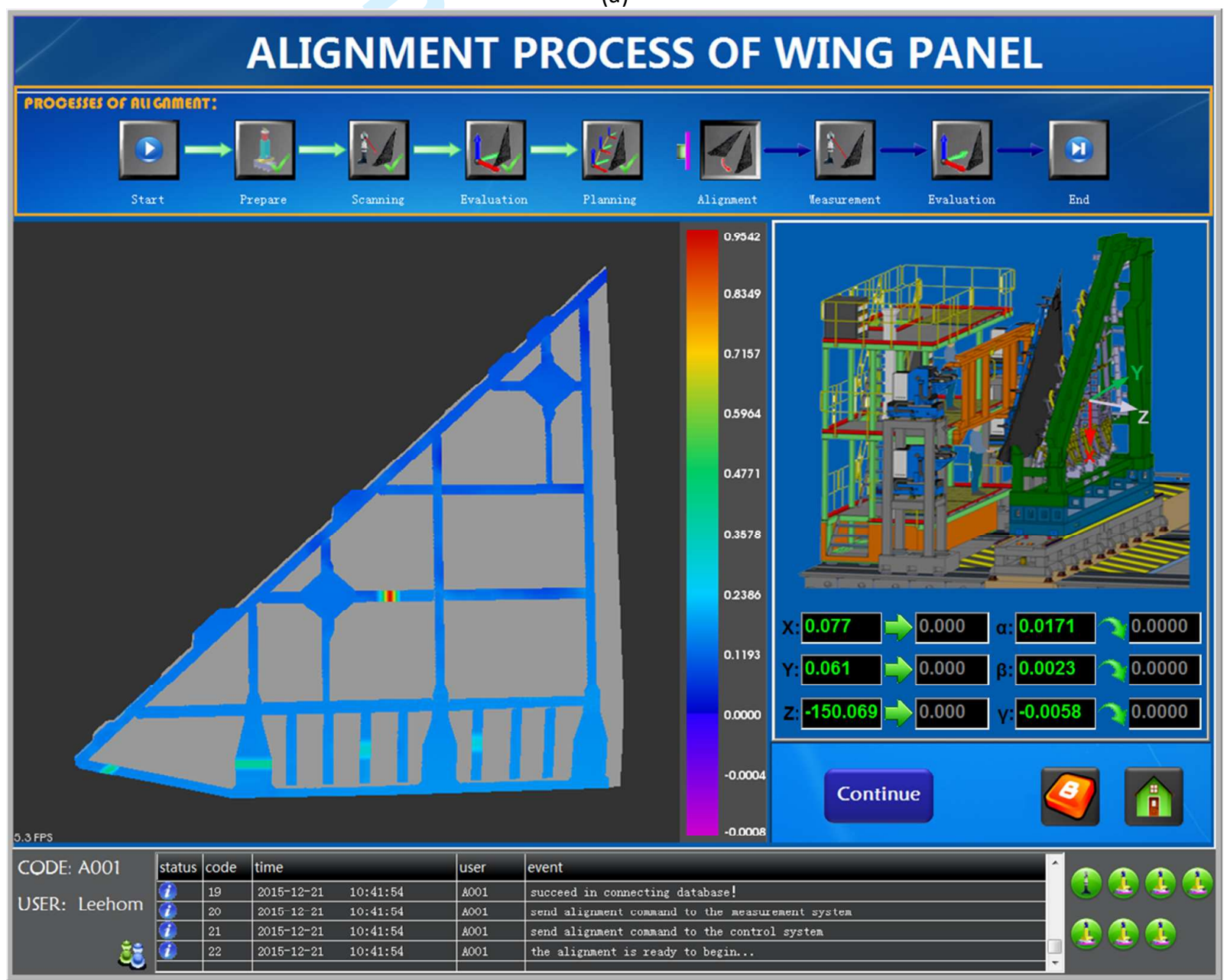

(b)

Figure 12 Gap map of mating surfaces

The overall gaps at 883,615 points are $68,127 \mathrm{~mm}$ when initially aligned, while the overall gaps are $38,349 \mathrm{~mm}$ when fine aligned as shown in figure 12 . The averaged gap reduced from $0.0771 \mathrm{~mm}$ to $0.0434 \mathrm{~mm}$. The extreme 
gaps at local regions A, B, C, D, E are out of required tolerances in figure 12(a), after the small posture transformation, they are controlled within tolerance as shown in figure 12(b). It is clearly showed that the assembly gaps are well optimized and controlled by the proposed method. Besides, some observations can be obatined that the deformation errors of the wing tips with acute angles are relatively large, this is because the panel is of large size and may deform at weak areas. From table 2, we can see that the further the point from the origin, the bigger the gap changes. It means that the gap changes are mainly caused by the rotations, which conforms with the fact that the components are usually rotated to adjust relative positions.

Figure 13 is the workshop where the wings are assembled. The wing panels are supported by the NCLs, and the understructures are positioned on the fixture as the assembly datum. Because the fighter airplane of new generation is involved in military secret, the wing product is forbidden to be shown in public, and the panel and understructure are removed in the picture. The proposed gap control method is based on posture alignment in automated assembly system by NCLs, thus it may not suitable for the traditional fixtures operated by humans.

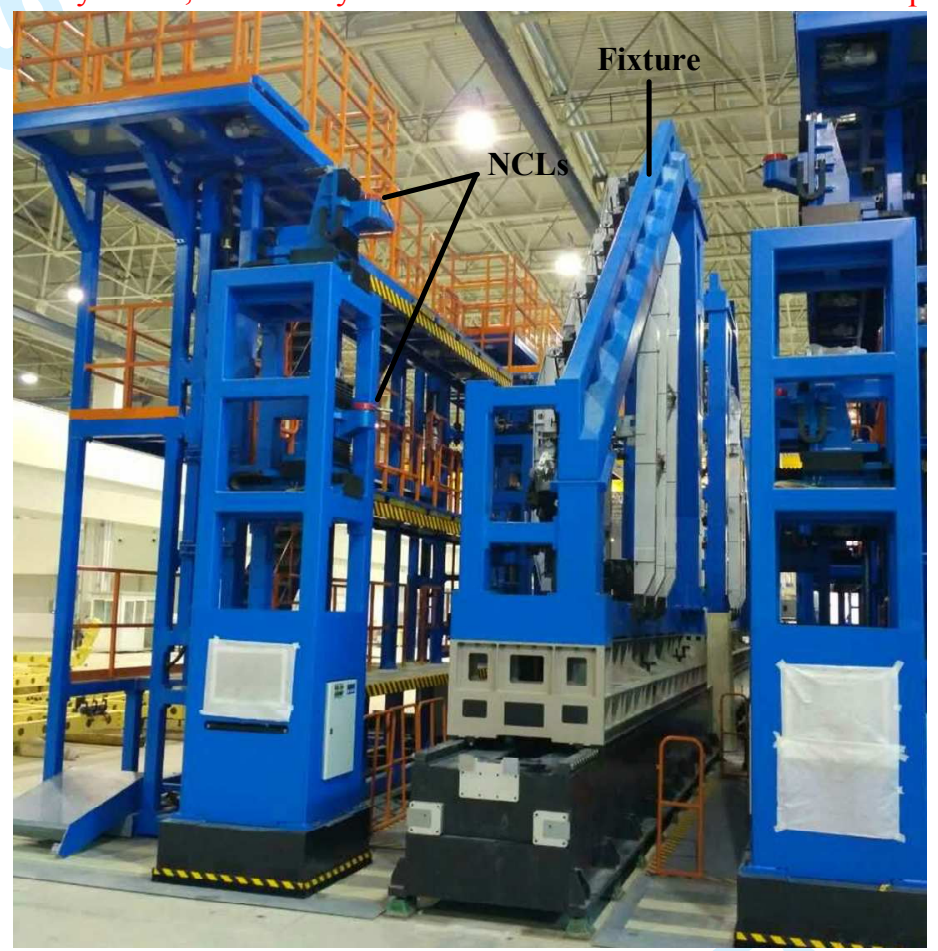

Figure 13 Assembly workshop

Due to the lack of published reference results, the proposed method can only be compared with the traditional method. On the conventional assembly line, the wing components are positioned on the fixtures, and then measured by thickness feelers manually. The adjustment process for gap control is to try best by the manual measurement feedback and adjustment operations, which consists of repeat measurement-adjustment-measurement. This is a complicated and cumbersome process, which should make sure that the assembly gaps are within tolerances to have good mechanical performance and ensure that the profile waviness is as small as possible to obtain better aerodynamic quality. According to the engineering practice, the manual adjustment process usually takes 3-5 hours. In some extreme conditions, the process will last for 1 2 days. However, with the proposed gap control method applied on the automated assembly line, the gap evaluation and posture adjustment can be implemented digitally and automatically, and the whole process costs within 30 minutes only.

\section{Conclusions}

Based on the strategy involving preliminary alignment and refined alignment, an optimal posture evaluation 
method is decomposed to initial posture estimation and final posture evaluation. A mathematical tool is introduced based on Small Posture Transformation (SPT), which linearizes the trigonometric transformation and simplifies the optimization. With the SPT, the gaps are updated by adding the original and delta gaps, so that exhaustive closest point searching is avoided. A comprehensive weighted model, which takes the point position error and surface fitness error as objectives and the gap tolerances as constraints, is established. By solving the constrained nonlinear problem, the assembly gaps of wing components are optimally distributed and coordinated.

The proposed method overcomes the problem of a lack of key measurement points for components of low strength and large size. Rather than a repeated manual measurement-adjustment-measurement process, the assembly of a wing panel and its understructure is automatically realized in this method, and the assembly gap requirements are met. Although the proposed approach redistributes the gaps in geometry, the fitness errors between two components can only be coordinated but not eliminated.

Future work should be focused on these subjects: (1) establishing and solving new control model with both gap and other constrains, like coaxiality and parallelism, for different assembly objects (e.g. fuselage sections); (2) carrying out statistical analysis on the gap regularity and offering compensation suggestions for manufacturing departments; (3) developing accurate shimming method for the residual gaps and its mechanics validation.

\section{Appendix}

For any $3 \mathrm{D}$ vectors $\mathbf{u}=\left(u_{1}, u_{2}, u_{3}\right), \mathbf{v}=\left(v_{1}, v_{2}, v_{3}\right), \mathbf{w}=\left(w_{1}, w_{2}, w_{3}\right), \mathbf{u} \times \mathbf{v} \cdot \mathbf{w}=\mathbf{u} \cdot(\mathbf{v} \times \mathbf{w})$ can be obtained.

Proof:

$$
\begin{aligned}
\mathbf{u} \times \mathbf{v} \cdot \mathbf{w} & =\left(u_{2} v_{3}-v_{2} u_{3}, u_{3} v_{1}-v_{3} u_{1}, u_{1} v_{2}-v_{1} u_{2}\right) \cdot\left(w_{1}, w_{2}, w_{3}\right) \\
& =w_{1} u_{2} v_{3}-w_{1} v_{2} u_{3}+w_{2} u_{3} v_{1}-w_{2} v_{3} u_{1}+w_{3} u_{1} v_{2}-w_{3} v_{1} u_{2} \\
& =u_{1}\left(w_{3} v_{2}-w_{2} v_{3}\right)+u_{2}\left(w_{1} v_{3}-w_{3} v_{1}\right)+u_{3}\left(w_{2} v_{1}-w_{1} v_{2}\right) \\
& =\left(u_{1}, u_{2}, u_{3}\right) \cdot\left(w_{3} v_{2}-w_{2} v_{3}, w_{1} v_{3}-w_{3} v_{1}, w_{2} v_{1}-w_{1} v_{2}\right) \\
& =\mathbf{u} \cdot(\mathbf{v} \times \mathbf{w})
\end{aligned}
$$

\section{References:}

Arun K. S., Huang T. S. and Blostein S. D. (1987). "Least-squares fitting of two 3-D point sets", IEEE Transactions On Pattern Analysis and Machine Intelligence, Vol.9, pp.698-700.

Andrew W. Fitzgibbon. (2003). "Robust registration of 2D and 3D point sets", Image and Vision Computing, Vol. 21, pp.1145-1153.

Almhdie A., Léger C., Deriche M. and Lédée R.. (2006). "A new implementation of the ICP algorithm for 3D surface registration using a comprehensive look up matrix", 14th European Signal Processing Conference (EUSIPCO 2006). Florence, Italy, September 4-8, 2006.

Besl, P. J., and Mckay, N. D. (1992). "A Method for Registration of 3D Shapes", IEEE Transactions on Pattern Analysis and Machine Intelligence, Vol.14, No.2, pp.239-256.

Birgin E.G., Castillo R.A., and Martlnez J.M. (2005). "Numerical Comparison of Augmented Lagrangian Algorithms for Nonconvex Problems", Computational Optimization and Applications, Vol.31, No.1, pp.31-55.

Cheng, L., Wang, Q., Li, J.X. and Ke, Y.L. (2014). "A posture evaluation method for a large component with thermal deformation and its application in aircraft assembly", Assembly Automation, Vol.34, No.3, pp.274-284.

Chen Zhehan, Du Fuzhou and Tang Xiaoqing. (2015). "Position and orientation best-fitting based on deterministic theory during large-scale assembly", Journal of Intelligent Manufacturing, pp.1-11. doi: 10.1007/s10845-015-1132-7. 
Felus Y.A. and Schaffrin B. (2005). "Performing similarity transformations using the error-in-variable model", Proceedings of ASPRS 2005 Annual Conference, pp. 220-227, Brentwood, Mo., USA.

Gregory C. Sharp, Sang W. Lee and David K. Wehe. (2002). "ICP Registration Using Invariant Features", IEEE Transactions on Pattern Analysis and Machine Intelligence, Vol.24, No.1, pp.1-25.

Horn B. K. P., Hilden H. M. and Negahdaripour S. (1988). "Closed-form solution of absolute orientation using orthonormal matrices", Journal of the Optical Society of America A, Vol.5, No.7, pp.1127-1638.

Helmut Pottmann, Martin Peternell and Bahram Ravani. (1999). "An introduction to line geometry with applications", Computer-Aided design, Vol.31, No.1, pp.3-16.

Jamshidi J., Kayani A., Iravani P., Maropoulos P. G. and Summers M. D.. (2010). "Manufacturing and assembly automation by integrated metrology systems for aircraft wing fabrication", Proceedings of the Institution of Mechanical Engineers, Part B: Journal of Engineering Manufacture, Vol.224, No.1, pp.25-31.

Nocedal Jorge and Wright Stephen J. (2006). "Numerical Optimization" (2nd ed.), Berlin, New York: Springer-Verlag.

Philip J.Schneider and David H.Eberly. (2005). "Geometric Tools for Computer Graphics", the Morgan Kaufmann series in computer graphics and geometric modeling.

Qian Xiaoping, Robinson Dean M., and Ross Joseph. (2005). "Admissible transformation volume for part dimensional quality gauging", Computer-Aided Design, Vol.37, No.13, pp.1335-1352.

Smith, Jamie M. (2011). "Concept Development of an Automated Shim Cell F-35 Forward Fuselage Outer Mold Line Control", Master Thesis, Manufacturing Engineering, University of Wisconsin-Stout.

Wilma Polini, M. Marrocu, and L. D'Ambrosio. (2007). "Tolerance analysis of free-form surfaces in composite material”, Journal of Computing and Information Science in Engineering, Vol.7, No.1, pp.31-43.

Yu Cijun, Li Jiangxiong, Yu Fengjie and Ke Yinglin. (2010). "3D point registration algorithm with engineering constraints", Journal of Mechanical Engineering, Vol.45, No.5, pp.183-190.

Zheng Lianyu, Zhu Xusheng, Liu Renwei, Wang Yiwei and Maropoulos P. G.. (2013). "A novel algorithm of posture best fit based on key characteristics for large components assembly", Procedia CIRP, Vol.10, pp.162-168. 